\title{
Bedside Clinical Assessment of Consciousness Level in Patients with Severe Chronic Disorders of Consciousness
}

\author{
Jun Shinoda*, Noriko Usami, Yoshitaka Asano and Yuka Ikegame \\ Chubu Medical Center for Prolonged Traumatic Brain Dysfunction, Kizawa Memorial Hospital and Department of Clinical Brain Sciences, Gifu University Graduate School \\ of Medicine, Minokamo, Gifu, Japan
}

\begin{abstract}
Conventional medical terms, vegetative state (VS) and minimally conscious state (MCS), for representing consciousness level of chronic disorders of consciousness (DOC) have two problems to use at bedside. One is a nosological issue, and the other is an issue related to scientific usability for clinical assessment. A three-by-two-graded classification, the chronic DOC scale (CDOCS), without any nosologically unfavorable and equivocal words, was established. The consciousness level of 143 patients with severe chronic DOC both at the time of admission and 24 months after the admission were determined using the VS/MCS classification and CDOCS, and the differences of the results of evaluation for improvement between these were assessed. Forty-seven (33\%) and $63(44 \%)$ patients were determined as having improved 24 months after admission on the VS/MCS classification and CDOCS, respectively. There was a significant difference in the percentages between these grading systems $(p<0.0001)$. The advantages of CDOCS are that it makes it possible to avoid using uncomfortable and ambiguous terms and that it is superior for evaluating nuanced consciousness level. We recommend the use of CDOCS for bedside assessment of consciousness level in patients with severe chronic DOC as a supplement for the conventional descriptions.
\end{abstract}

Keywords: Chronic disorders of consciousness; Vegetative state; Minimally conscious state; Grading of consciousness level; Bedside clinical assessment

\section{Introduction}

In Japan, the number of patients with chronic disorders of consciousness (DOC) was presumed to be about 3,000 in 1978 according to a field assessment of the Tohoku region [1]. The number of such patients in 2010, however, was estimated to be about 55,000 [2]. These data suggest an 18 -fold increase in number in chronic DOC over 32 years. In the United States, the number of individuals who sustain severe Traumatic Brain Injury (TBI) with prolonged loss of consciousness each year is estimated to be between 56 and 170 per million [3]. Disappointingly, there has been no epoch-making treatment for improving the disturbed consciousness in these patients. Therefore, the increased number of these patients has become a serious issue not only medically, but also socially. One of the medical problems is that conventional medical terms such as vegetative state (VS) [4,5] and minimally conscious state (MCS) [6] for representing consciousness level of chronic DOC are not considered satisfactory by many bedside assessors.

There are two irreconcilable problems with using this VS/MCS classification. One is the nosological issue; the terms "vegetative" and "minimally conscious" sound uncomfortable, ambiguous, and border on derogatory. The second issue is related to scientific usability for clinical assessment; it is hard to evaluate nuance of consciousness level at bedside. The two-graded classification system using VS and MCS is too simplistic for evaluating nuance of consciousness levels.

Recently, sub-categorization of MCS into MCS+ and MCS- based on the level of complexity of observed behavioral responses was proposed, in which MCS+ was defined by the presence of command-following, intelligible verbalization, or gestural or verbal yes/no responses [7]. The new three-graded classification system after adding MCS+/- may be preferable to the current system. However, expert medical staffs who are treating patients with severe chronic DOC everyday need an intuitive, easy-to-use, yet detailed and concise classification system for describing impaired consciousness at the bedside.

The Coma Recovery Scale-Revised (CRS-R) has excellent content validity and test-retest reliability, and is recommended for use in detailed evaluation of chronic DOC [8]. However, the reliability of this scale depends on examiner experience in applying the scale. The learning-curve burden to the assessor might negatively impact compliance, causing the assessor not to use the scale during routine clinical work at bedside. Additionally, in the CRS-R, because substantial scoring weight depends on auditory, visual, and motor functions, which are factors not directly affected by consciousness impairment, the base level consciousness may sometimes be underestimated or overestimated.

In consideration of these issues, we have established an original three-by-two-graded classification system for diagnosing severe chronic DOC, which is called the chronic DOC scale (CDOCS). We have been using the prototype of this scale, the Communication Grading System for Prolonged Consciousness Impairment, which had established in our hospital, for the past 10 years [9]. CDOCS, which is a modified scale of the prototype, is simple and easy to use without any nosologically unfavorable and equivocal words. In this study, we examined whether the CDOCS can supplement the VS/MCS classification system in terms of evaluation of nuanced consciousness level in patients with severe chronic DOC.

\section{Materials and Methods}

\section{Patients}

Our hospital, the Chubu Medical Center for Prolonged Traumatic

*Corresponding author: Jun Shinoda, MD, PHD, IFAANS, FRCFED, Chubu Medical Center for Prolonged Traumatic Brain Dysfunction, Kizawa Memorial Hospital and Department of Clinical Brain Sciences, Gifu University Graduate School of Medicine, 630 Shimo-kobi, Kobi-cho, Minokamo, 505-0034 Gifu, Japan, Tel: +81-574-24-2233; Fax: +81-574-24-2230; E-mail: junshino@joy.ocn.ne.jp

Received October 15, 2015; Accepted November 02, 2015; Published November 09,2015

Citation: Shinoda J, Usami N, Asano Y, Ikegame Y (2015) Bedside Clinical Assessment of Consciousness Level in Patients with Severe Chronic Disorders of Consciousness. Int J Neurorehabilitation 2: 186. doi:10.4172/2376-0281.1000186

Copyright: (c) 2015 Shinoda J, et al. This is an open-access article distributed under the terms of the Creative Commons Attribution License, which permits unrestricted use, distribution, and reproduction in any medium, provided the original author and source are credited. 
Brain Dysfunction, Kizawa Memorial Hospital, is a specialized institution for the medical therapy and care of patients with severe chronic DOC after traffic accident related traumatic brain injury. From when we opened our doors in 2001 until April 2014, we have treated 217 inpatients. All patients are classified on the Glasgow Outcome Scale (GOS) [10] at the time of admission with persistent vegetative state or severe disability with impaired consciousness. Our treatment protocol includes rehabilitation, medication, nursing care and, occasionally, surgical intervention, such as cerebrospinal fluid diversion and intrathecal Baclofen infusion. The term of admission is $2-3$ years.

Of our 217 patients, 163 were admitted between January 2005 and January 2012. Of the 163 patients, 4 died of pneumonia during admission. Of the remaining 159 patients, 143 who were admitted between 5 and 24 months after the accident were enrolled in this bedside clinical assessment of chronic DOC. The remaining 16 patients were not enrolled in this study because they were admitted to our hospital in an acute/subacute phase (within 3 months after traffic accident) or their admission periods were less than 24 months. The mean age of the enrolled 143 patients was 36.5 years with a standard deviation of \pm 16.8 years. The cohort included 36 women. The mean interval from the accident to the admission was 8.2 months. The main causes of chronic DOC were TBI including severe diffuse brain injury, cerebral contusion, traumatic cerebral hematoma, and subdural hematoma in 139 patients, and hypoxic encephalopathy due to head injury in 4 patients. This study was approved by the Ethics Committee of the Kizawa Memorial Hospital.

\section{Chronic DOC scale}

In CDOCS, Level I includes patients who can communicate by language or gestures even if it is minimal, where communication is defined as command-following, basic greeting exchanges, yes/no response, and/or that using instruments like a talking aid or a character board. Level II includes patients who cannot communicate verbally or by gestures, but who respond with more than simple reflexes to external stimuli. This includes movement with aim, ocular pursuit, eye contact and change of facial expression, i.e., they can appropriately recognize their environment. Level III includes patients who cannot respond with more than a reflex to any external stimuli, but who may exhibit involuntary movement, flexion/extension of the limbs in response to pain, spontaneous eye opening, muscle contraction, change of respiration pattern, coughing, or yawning.

Levels I and II are divided into two sub-levels A and B, according to the presence or absence of reproducibility/promptness of response. The presence of reproducibility/promptness of response means that patients can always communicate verbally or respond to external stimuli within several seconds consistently. Level III is also divided into two sub-levels A and B according to the presence or absence of aimless spontaneous tiny movements including flexion/extension of the fingers and limbs and rotation of the head which are neither reflexes nor pathologic involuntary movements such as tremor, tic, convulsion, and shivering (Figure 1).

\section{Assessment}

The consciousness level of the patients both at the time of admission and 24 months after the admission were determined using

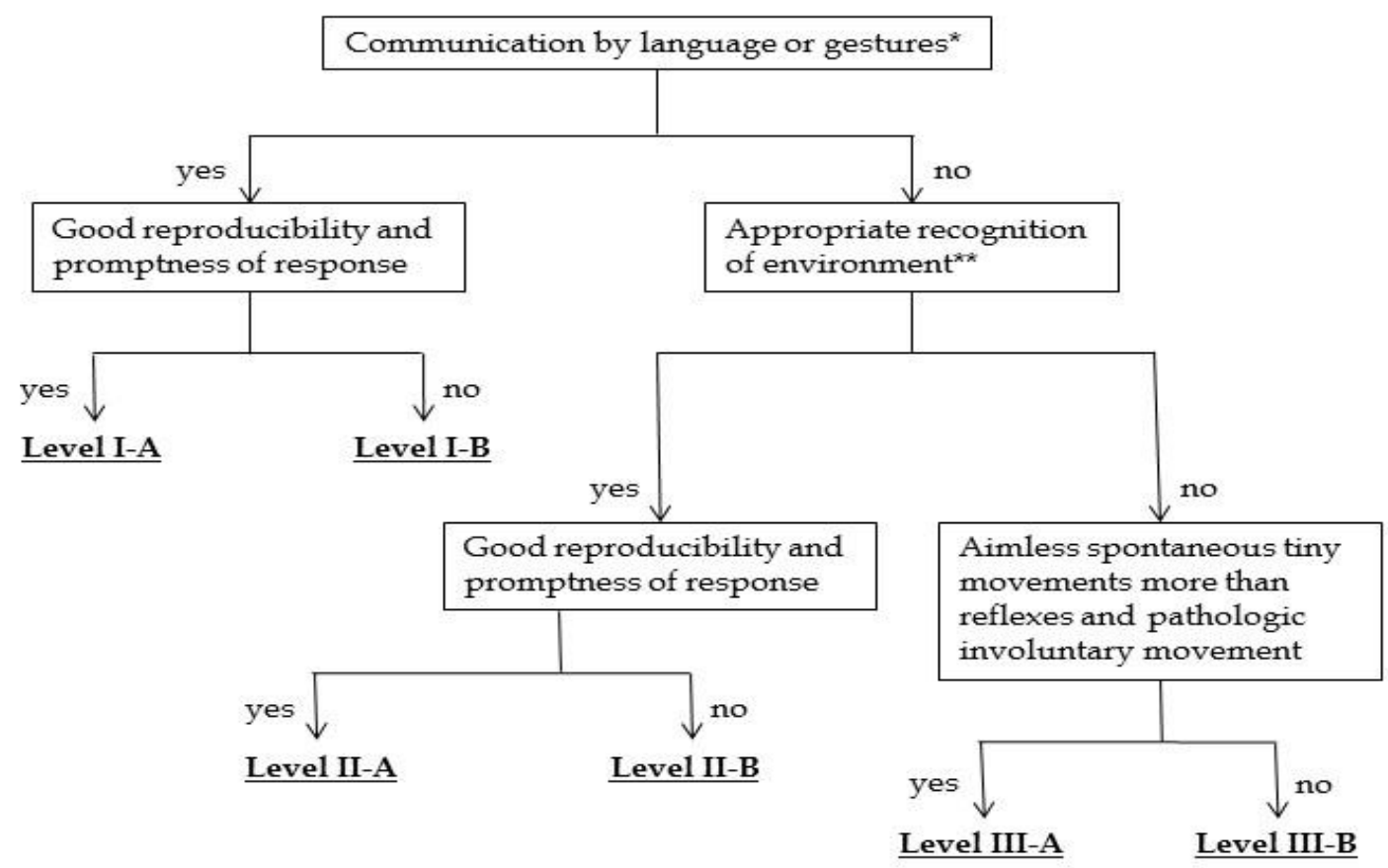

Figure 1: Suggested process for diagnosing the severity of impaired consciousness in patients with severe chronic disorders of consciousness in the Chronic DOC Scale (CDOCS).

*Whether patients can communicate by language or gestures even if it is minimal, where communication is defined as command-following, basic greeting exchanges, yes/no response, and/or that using instruments like a talking aid or a character board.

**Whether patients respond with more than simple reflexes to external stimuli. This includes movement with aim, ocular pursuit, eye contact and change of facial expression, i.e. they can appropriately recognize their environment 
both the VS/MCS classification system and CDOCS with the consensus of 3 neurosurgeons and 3 senior nurses. The consciousness level of each patient was determined based on the detailed description of the results of neurological examinations performed every two weeks in the clinical record during the admission, retrospectively. The clinical states of patients of VS, MCS-, and MCS+ were determined according to the definitions described in the reports by American Medical Association Council on Scientific Affairs and Council of Ethical and Judicial Affairs [5], Giacino et al. [6], and Bruno et al. [7].

Briefly, among chronic DOC, MCS is characterized by inconsistent but clearly discernible behavioral evidence of consciousness and can be distinguished from coma and VS by documenting the presence of specific behavioral features not found in either of these conditions [6]. To make the diagnosis of MCS, limited but clearly discernible evidence of self or environmental awareness must be demonstrated on a reproducible or sustained basis by one or more of the following behaviors: following simple commands, gestural or verbal yes/no responses (regardless of accuracy), intelligible verbalization, and purposeful behavior, including movements or affective behaviors that occur in contingent relation to relevant environmental stimuli and are not due to reflexive activity [6]. MCS+ was defined by the presence of command-following, intelligible verbalization, or gestural or verbal yes/no responses among MCS [7].

The differences of the results of evaluation for improvement between these grading systems were assessed. Patients whose consciousness was improved to the level of severe disability with full conscious state or better with a GOS score 24 months after the admission were determined as having emerged from DOC.

\section{Results}

The number of patients assessed as VS, MCS-, and MCS+ at the time of admission were 62, 28, and 53, respectively. At 24 months post-admission, $17(27 \%), 12(43 \%)$, and $18(34 \%)$ of them were determined to have showed improvement. Overall, 47 patients (33\%) were determined as having improved in consciousness on the VS/ MCS classification system (Table 1). The number of patients in Levels III-B, III-A, II-B, II-A, I-B, and I-A of CDOCS at the time of admission was $49,13,14,14,26$, and 27 , respectively. At the same interval of 24 months post-admission, 13 (26\%), 7 (54\%), 8 (57\%), 7 (50\%), 16 (62\%), and 12 (44\%) of them were determined as having improved. Overall, 63 patients (44\%) were determined as having improved in consciousness on CDOCS (Table 2). Sixteen patients who were determined as having not improved on the VS/MCS classification system were determined as having improved on CDOCS. There was a significant difference of the percentages of patients determined as having improved in consciousness from the VS/MCS classification system to CDOCS $(p<0.0001$, chi-square test) (Table 3 ).

\section{Discussion}

For decades, conventional medical terms such as VS $[4,5]$ and MCS [6] have been used for clinical diagnosis of patients with severe chronic $\mathrm{DOC}$ at the bedside. However, there are two irreconcilable issues with the VS/MCS classification schema. One is a nosological issue. The word "vegetative" has an unintended negative connotation and a pejorative and stigmatic undertone. The word "minimally conscious" sounds ambiguous; it is difficult for a layperson to form a concrete image of a patient with such a consciousness state, although the definition was clinically established. The European Task Force on DOC has recently proposed the more neutral term "unresponsive wakefulness syndrome" instead of the negative term VS [11]. We, however, sometimes experience patients with severe VS who have equivocal sleep-wake cycles, which can barely open their eyes spontaneously, or upon stimulation, and who exhibit no focusing, ocular pursuit, or blink reflex. The state of these patients cannot necessarily be accepted as being wakeful by laypersons. The other is an issue related to scientific usability for clinical assessment at bedside. It is hard to evaluate nuance of consciousness level at bedside using the VS/MCS classification.

CDOCS is fundamentally a grading system that assesses the patient's potential for interaction with the environment, including other persons. The key differentiating factor between Levels I and II is simply whether any communication by language or gestures can be observed; for Levels II and III it is whether any awareness of the environment can be observed. Level I mostly corresponds to MCS+, Level II to MCS- and Level III to VS.

In patients in Levels I and II of CDOCS, reproducibility and promptness of response to the external stimuli including alteration of the environment and calling from other persons are important factors to catch the subtle improvement of consciousness level at the bed side. However, these factors have not been included in any conventional evaluation system for chronic DOC without the auditory functional scale in CRS-R. CDOCS is unique because the evaluation weight substantially depends on factors of reproducibility and promptness of response. Thirteen $(81 \%)$ of the 16 patients who were not determined as having improved on the VS/MCS classification system, but were determined as having improved on CDOCS were patients who were determined as having improved in this point of view on CDOCS.

\begin{tabular}{|c|c|c|c|c|c|}
\hline At admission & \multicolumn{5}{|c|}{24 months after admission } \\
\hline (No. of patients) & VS & MCS- & MCS+ & Emerged & Improvement (\%) \\
\hline VS (62) & 45 & 9 & 8 & 0 & 27 \\
\hline MCS- (28) & 0 & 16 & 8 & 4 & 43 \\
\hline MCS+ (53) & 0 & 0 & 35 & 18 & 34 \\
\hline
\end{tabular}

*See description detailed in the text. VS=Vegetative State. MCS=Minimally Conscious State.

Table 1: Diagnostic results by VS/MCS classification system*.

\begin{tabular}{|c|c|c|c|c|c|c|c|c|}
\hline At admission & \multicolumn{10}{|c|}{24 months after admission } \\
\hline (No. of patients) & III-B & III-A & II-B & II-A & I-B & I-A & Emerged & Improvement (\%) \\
\hline III-B (49) & 36 & 3 & 5 & 2 & 1 & 2 & 0 & 27 \\
\hline III-A (13) & 0 & 6 & 1 & 1 & 1 & 4 & 0 & 54 \\
\hline II-B (14) & 0 & 0 & 6 & 3 & 3 & 0 & 2 & 57 \\
\hline II-A (14) & 0 & 0 & 0 & 7 & 4 & 1 & 2 & 50 \\
\hline I-B (26) & 0 & 0 & 0 & 0 & 10 & 10 & 6 & 62 \\
\hline I-A (27) & 0 & 0 & 0 & 0 & 0 & 15 & 12 & 44 \\
\hline
\end{tabular}

*See description detailed in the text. CDOCS=Chronic Disorder Of Consciousness Scale

Table 2: Diagnostic results by CDOCS*

\begin{tabular}{|c|c|c|c|}
\hline \multicolumn{5}{|c|}{ VS/MCS classification system } \\
\hline CDOCS & Unchanged & Improved & Total \\
\hline Unchanged & 80 & 0 & $80(55.9 \%)$ \\
\hline Improved & 16 & 47 & $63(44.1 \%)$ \\
\hline Total & $96(67.1 \%)$ & $47(32.9 \%)$ & $143(100 \%)$ \\
\hline
\end{tabular}

*There was a significant difference in the percentages of patients determined as improved in consciousness between the VS/MCS classification system and the CDOCS ( $p<0.0001$, chi-square test). VS=Vegetative State.

MCS=Minimally Conscious State. CDOCS=Chronic Disorder of Consciousness Scale.

Table 3: Comparison of the number of improved patients between VS/MCS classification system and CDOCS*. 
To check aimless spontaneous tiny movements more than reflexes and pathologic involuntary movement in the fingers, limbs, body, and/ or head is important to evaluate subtle brain activities in patients with chronic DOC. In patients of Level III, particularly, these movements are one of the few externally apparent signs indicating the presence of brain activities at bedside. At the very least, the brain activity of patients with such movements is estimated to be higher than that of patients without such movements. Actually, 3 (19\%) of the 16 patients who were not determined as having improved on the VS/MCS classification system, but were determined as having improved on CDOCS were patients who were determined as having improved in this regard using CDOCS.

The limitation of CDOCS is that it is inappropriate for patients with mild-moderate chronic DOC who can communicate verbally in a more-than-minimal facility. To assess precise neuropsychological function, neuropsychiatric examinations of higher precision are preferable.

The primary caregivers of patients with chronic DOC every day consist of medical staff such as nurses, nurse's aides, health-care technicians, rehabilitation therapists, and/or medical non-professionals such as family caregivers. They often have difficulties in talking about the diagnosis of such patients in bedside discussion because of the unfavorable terms such as VS. It seems difficult for laypersons to understand the meaning of MCS. The advantages of CDOCS are that it makes it possible to avoid using uncomfortable and ambiguous terms and that the grading criteria are easy to understand for every medical staff assessor in addition to the superiority for evaluating nuanced consciousness level at bedside.

\section{Conclusions}

CDOCS makes it possible to avoid using uncomfortable and ambiguous terms. Additionally, the results of this study showed that CDOCS is significantly better for evaluating nuance of consciousness level. We would recommend the use of this simple scale, CDOCS, for bedside assessment of consciousness level in patients with severe chronic DOC as a supplement for the conventional descriptions of VS and MCS, although further studies for confirming validation and reliability of this system are needed to use it widely in clinical setting.

\section{Conflicts of Interest and Disclosure}

The authors declare that we have no conflict of interest relevant to the content of this article.

\section{References}

1. Sato S, Ueki K, Arai H, Suzuki J, Kodama N, et al. (1978) Epidemiological survey of vegetative state patients in Tohoku district in Japan. Neurol Med Chirur (Tokyo) 18: 141-145.

2. Fujiwara S (2011) A survey of patients with prolonged impairment of consciousness in Miyagi prefecture. Proceeding of the 20th Annual Meeting of the Japan Coma Society, p41

3. Laureys S (2007) Eyes open, brain shut. Sci Am 296: 84-89.

4. Teasdale G, Jennett B (1974)Assessment of coma and impaired consciousness. A practical scale. Lancet 2: 81-84.

5. Persistent vegetative state and the decision to withdraw or withhold life support. Council on Scientific Affairs and Council on Ethical and Judicial Affairs. JAMA 263: 426-430.

6. Giacino JT, Ashwal S, Childs N, Cranford R, Jennett B, et al. (2002) The minimally conscious state: definition and diagnostic criteria. Neurology 58: 349 353

7. Bruno MA, Vanhaudenhuyse A, Thibaut A, Moonen G, Laureys S (2011) From unresponsive wakefulness to minimally conscious PLUS and functional locked-in syndromes: recent advances in our understanding of disorders of consciousness. J Neurol 258: 1373-1384.

8. Giacino JT, Kalmar K, Whyte J (2004) The JFK Coma Recovery Scale-Revised: measurement characteristics and diagnostic utility. Arch Phys Med Rehabil 85 2020-2029.

9. Shinoda J, Asano $Y$ (2009) Evaluation and management of coma patients Essential Practice of Neurosurgery. In Kalangu KKN, Kato Y, Dechambenoit G (ed), Access Publishing, Nagoya, pp51-66.

10. Jennett B, Bond M (1975) Assessment of outcome after severe brain damage. Lancet 1: $480-484$

11. Laureys S, Celesia GG, Cohadon F, Lavrijsen J, León-Carrión J, et al. (2010) Unresponsive wakefulness syndrome: a new name for the vegetative state of apallic syndrome. BMC Med 8: 68. 